\section{BMJ}

Open

Gastroenterology

\title{
Primary hepatic large B-cell lymphoma following direct-acting antiviral treatment for hepatitis C
}

\author{
Yosra Zaimi, ${ }^{1}$ Myriam Ayari (10 , , ${ }^{1}$ Walid Cherifi, ${ }^{2}$ Feryel Letaief Ksontini, ${ }^{3}$ \\ Shema Ayadi, ${ }^{1}$ Emna Bel Hadj Mabrouk, ${ }^{1}$ Myriam Jrad $^{2}$
}

To cite: Zaimi Y, Ayari M, Cherifi W, et al. Primary hepatic large B-cell lymphoma following direct-acting antiviral treatment for hepatitis C. BMJ Open Gastro 2021;8:e000721. doi:10.1136/ bmjgast-2021-000721

Received 8 June 2021 Accepted 1 August 2021
Check for updates

(C) Author(s) (or their employer(s)) 2021. Re-use permitted under CC BY-NC. No commercial re-use. See rights and permissions. Published by BMJ.

${ }^{1}$ Hepato-gastroenterology, Charles Nicolle Hospital, Tunis, Tunisia

${ }^{2}$ Radiology, Charles Nicolle Hospital, Tunis, Tunisia ${ }^{3}$ Medical Oncology, Institut Salah-Azaïz, Tunis, Tunisia

Correspondence to Dr Myriam Ayari; ayari.myriam@hotmail.fr

\section{ABSTRACT}

Hepatitis $\mathrm{C}$ virus (HCV) infection is a major public health concern worldwide, raising important medical and economic issues. HCV-related end-stage liver disease is one of the most common indications for hepatic transplantation. Chronic hepatitis $\mathrm{C}$ is also assimilated to a systemic disease because of multiple extrahepatic manifestations, including lymphoproliferative disorders. The revolution of HCV treatment with the advent of direct-acting antivirals has significantly improved the management with high antiviral efficacy and good safety profile compared with old regimens, thus allowing good outcomes on hepatic and extrahepatic symptoms. However, with the widespread use of these new agents, controversial concerns about unexpected increasing cases of hepatocellular carcinoma were reported. We now report the case of a patient presenting with HCV-related cirrhosis, treated with direct-antiviral therapy and diagnosed with primary hepatic lymphoma shortly after the end of the treatment.

\section{INTRODUCTION}

Hepatitis $\mathrm{C}$ virus (HCV) is a lymphotropic, hepatotropic virus and one of the major causes of chronic liver disease. In addition to the risk of progression to cirrhosis and its related complications, HCV exposes also to extrahepatic manifestations such as lymphoproliferative disorders ranging from benign to aggressive lymphoma, leading to significant morbidity and mortality. Currently, there is no effective vaccine against HCV. Nevertheless, the development of direct-acting antivirals (DAAs) has revolutionised the treatment of hepatitis $\mathrm{C}$ with sustained virological response (SVR) rates $>90 \%$ in all genotypes. Viral eradication can prevent, stabilise or even regress liver fibrosis lesions and portal hypertension, thus reducing chronic liver disease complications. Besides, antiviral treatment may allow the regression of extrahepatic manifestations related to HCV. This revolution in the management of patients with chronic hepatitis $\mathrm{C}$ has nurtured, consequently, the hope that hepatic carcinogenesis risk would significantly decrease. Yet, concerns were expressed, noting increasing reported cases of hepatocellular carcinoma (HCC) after antiviral treatment, thus generating a long debate these recent years. However, there are very scant reports regarding hepatic lymphoma, an uncommon liver tumour, occurring after viral clearance with DAAs. Herein, we report a case of patient with HCV-related cirrhosis, treated successfully with sofosbuvir/ribavirin regimen and developing a primary hepatic lymphoma shortly thereafter.

\section{CASE PRESENTATION}

A 58-year-old Caucasian man was diagnosed with Child A5 cirrhosis secondary to chronic hepatitis C genotype 2 infection in August 2019. Medical history included only epilepsy well controlled with lamotrigine. Family medical history was irrelevant. The patient did not take any other medications and did not consume any alcohol, herbs or toxics. Laboratory findings showed normal haemoglobin of $136 \mathrm{~g} / \mathrm{L}$, normal platelet level, normal albumin level and hypocholesterolaemia of $3.65 \mathrm{mmol} / \mathrm{L}$. Hepatic tests indicated only elevated transaminases-aspartate aminotransferase of $60 \mathrm{U} / \mathrm{L}$ and alanine aminotransferase of $490 \mathrm{U} / \mathrm{L}-$ and slightly elevated international normalised ratio of 1.3 (normal range 0.8-1.2). There was no cholestasis. Laboratory blood analysis results are summarised in table 1 . His fibroscan showed a median of $13 \mathrm{kPa}$, correlating to a fibrosis score of F4. On upper endoscopy, the patient presented grade 2 oesophageal varices and gastric varices GOV II. Primary prophylaxis of variceal bleeding was initiated with propranolol. The viral load (HCV RNA) was $1.7 \times 10^{5}$ $\mathrm{UI} / \mathrm{mL}$. Abdominal ultrasound performed just before antiviral therapy showed dysmorphic liver without focal lesions and collateral circulation. DAA therapy based on 24 weeks 


\begin{tabular}{lcc}
\hline Table $1 \quad$ Laboratory blood test results & \\
\hline Blood test & Result & $\begin{array}{l}\text { Reference } \\
\text { range }\end{array}$ \\
\hline White blood cell count $\left(/ \mathrm{mm}^{3}\right)$ & 4300 & $4000-10000$ \\
\hline Neutrophils $\left(/ \mathrm{mm}^{3}\right)$ & 1849 & $1500-7000$ \\
\hline Lymphocytes $\left(/ \mathrm{mm}^{3}\right)$ & 2150 & $1500-4000$ \\
\hline Monocytes $\left(/ \mathrm{mm}^{3}\right)$ & 172 & $200-800$ \\
\hline Eosinophils $\left(/ \mathrm{mm}^{3}\right)$ & 129 & $<400$ \\
\hline Basophils $\left(/ \mathrm{mm}^{3}\right)$ & 0 & $<100$ \\
\hline Red blood cell count (millions/ & 4.3 & $4.2-5.7$ \\
mm³) & 139 & $13-17$ \\
\hline Haemoglobin (g/L) & 41 & $40-52$ \\
\hline Haematocrit (\%) & 169 & $150-400$ \\
\hline Platelets (billion/L) & 150 & $140-280$ \\
\hline Lactate dehydrogenase (U/L) & 36 & $35-45$ \\
\hline Albumin (g/dl) & 72 & $60-80$ \\
\hline Total protein (g/L) & 3.65 & $4.14-5.18$ \\
\hline Cholesterol (mmol/L) & 60 & $70-100$ \\
\hline Prothrombin time (\%) & 60 & $<40$ \\
\hline $\begin{array}{l}\text { Aspartate aminotransferase } \\
\text { (U/L) }\end{array}$ & & \\
\hline Alanine aminotransferase (U/L) & 490 & $<40$ \\
\hline $\begin{array}{l}\text { Gamma-glutamyl } \\
\text { transpeptidase (U/L) }\end{array}$ & 41 & $10-50$ \\
\hline Alkaline phosphatase (IU/L) & 92 & $40-130$ \\
\hline Total bilirubin (mg/dL) & 10 & $0.3-1.2$ \\
\hline & &
\end{tabular}

of sofosbuvir and ribavirin regimen was initiated on August 2019. Serum HCV RNA levels rapidly decreased, and SVR was successfully achieved without any adverse events. As part of HCC screening, ultrasound was repeated by the end of January 2020, shortly after the end of antiviral treatment, revealing many hypoechogenic nodules mainly in the right liver lobe. The patient was asymptomatic. On physical examination, there was only hepatomegaly without evidence of ascites, and peripheral lymph nodes were not enlarged. Multifocal HCC or hepatic metastases were suspected and thoraco-abdomino-pelvic CT scan was performed, detecting multiple lesions (figure 1) arising from the right hepatic lobe, of which the largest was in segment VII measuring $42.5 \times 35 \mathrm{~mm}$. Enhancement of these lesions was heterogeneous after contrast injection without arterial hypervacularisation or washout. There was no evidence of any primary tumour, adenopathies or other pathological findings. The patient, then, underwent a contrast MRI of the abdomen, confirming the presence of multiple nodules mainly in the right lobe. These lesions were hyperintense in T2-weighted imaging and hypointense in T1-weighted imaging without contrast enhancement on the arterial phase and with hypointense appearance on the portal phases (figure 2). These features ruled out HCC typical behaviour. Upper endoscopy and colonoscopy done while investigating malignancy showed no potential digestive tumour. Tests for serum

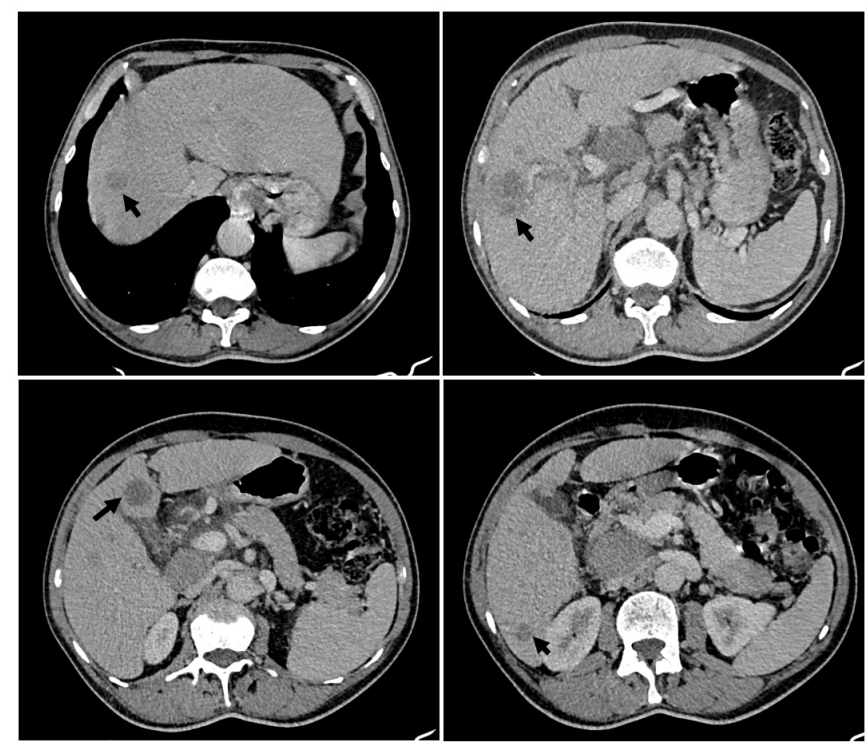

Figure 1 Abdominal CT scan views showing dysmorphic liver with lobulated contours and multiple nodular hepatic lesions of different sizes (arrows).

tumour markers including alpha-fetoprotein, carcinoembryonic antigen and cancer antigen 19-9 showed normal values. As imaging, laboratory and endoscopic work-up were unremarkable, it was decided to perform ultrasound-guided biopsy. After a first attempt showing only fibroinflammatory lesions suggesting pseudo-inflammatory tumour, percutaneous core-needle biopsy was repeated a second time. Histological examination with immunohistochemical study concluded a large B-cell lymphoma with positive CD20 staining and negative CD5 and CD3 (figure 3). The proliferation factor measured by Ki67 was very high. Gene mutation study indicated non-double-hit profile (BCL2+/ MYC-). Position emission tomography scan could not be performed due to difficulties in accessibility and being unavailable in our institution. As part of the disease assessment, bone marrow biopsy and lumbar punction were performed and excluded bone marrow and central nervous system affection. Serological markers of Epstein-Barr virus, herpes simplex virus and HIV were negative. The patient presented with good performance status (PS1) and thus received six cycles of immunochemotherapy with R-CHOP (rituximab-cyclophosphamide, doxorubicin, vincristine and prednisone). Follow-up imaging after treatment has shown partial response of the disease according to the CHESON criteria, characterised by a decrease in the target tumour volume estimated at $77 \%$ with appearance of a large amount of ascites. There was no newly appearing suspicious lesion.

\section{DISCUSSION}

Hepatitis $\mathrm{C}$ is a major public health concern throughout the world, exposing the patient to possible complications related to portal hypertension and liver failure, as well as to the risk of malignancy and extrahepatic manifestations. Recently, the development of new direct antiviral molecules directed against the three major HCV targets (NS3/4A protein, NS5B protein and NS5A protein) has 


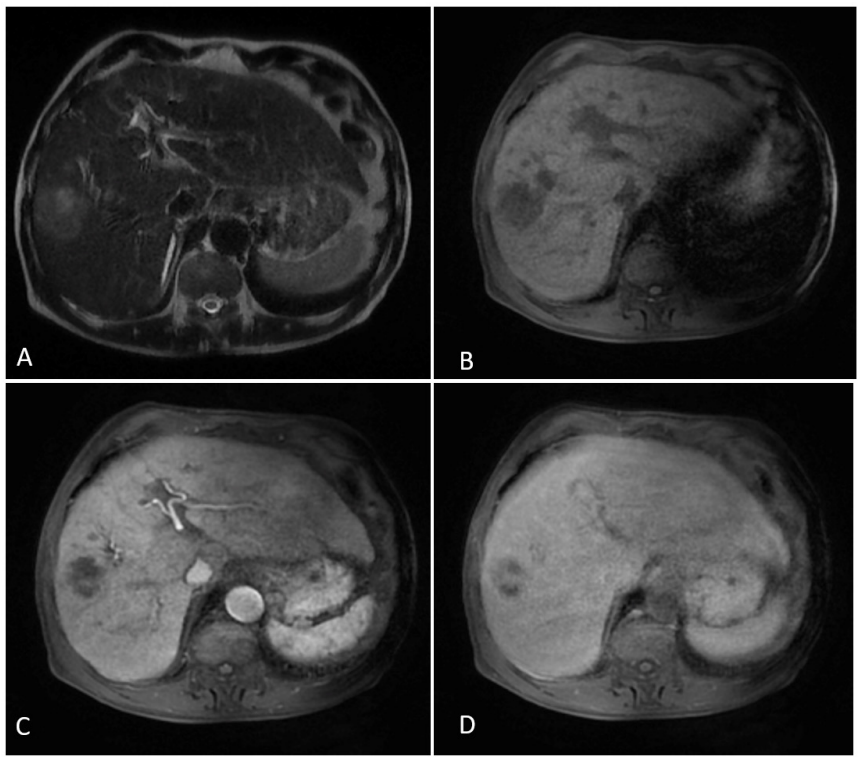

Figure $2 \mathrm{MRI}$ axial sequences centred on the largest lesion. Nodule straddling segments $\mathrm{VI}$ and VII of the liver showing heterogeneous hypersignal on T2-weighted image $(A)$ and spontaneous hyposignal on T1-weighted image (B) without washin at the arterial time $(\mathrm{C})$ or washout at the portal time (D).

significantly improved the management of chronic hepatitis C with short 'interferon-free' treatment regimens that provide an SVR of up to $90 \%-100 \%$ and few adverse effects.

Primary hepatic lymphoma (PHL) occurring after antiviral therapy is rather uncommon and only discussed in minimal previous reports in the literature. PHL is a very rare malignancy and reported as case reports in
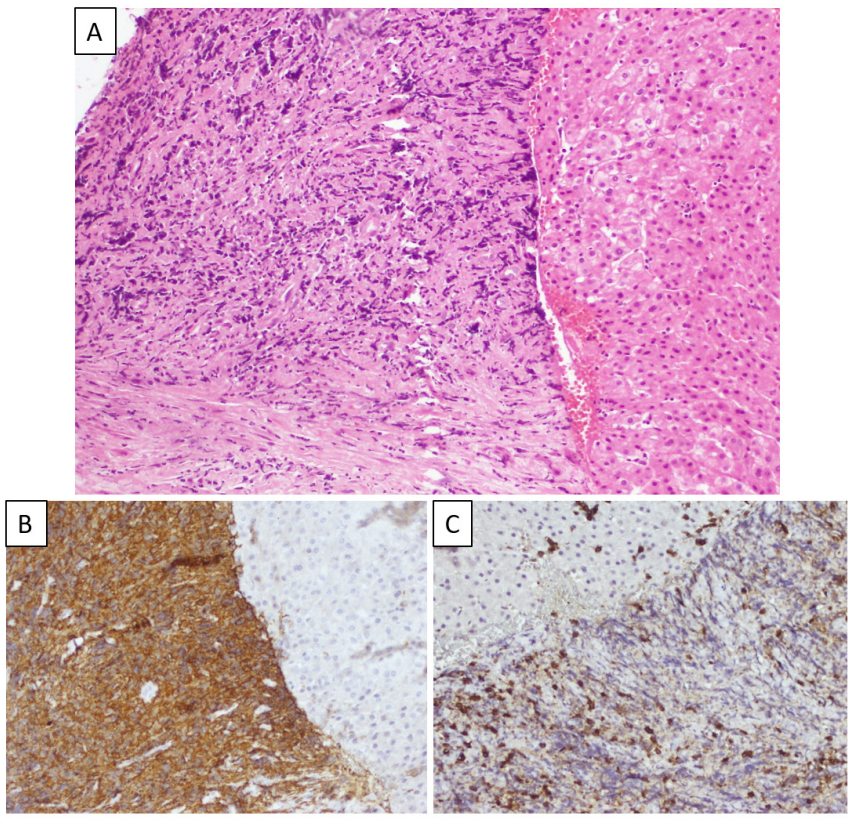

Figure 3 Liver tumour histopathology. H\&E examination at $\times 200$ magnification showing dense B-cell lymphoepithelial infiltration of hepatic parenchyma (A). Immunochemistry staining revealing positive CD20 (B) and negative CD5 (C). the literature, mostly corresponding to large B-cell type. There are even few available data on PHL occurring after DAA therapy, and to the best of our knowledge, there are only two cases reporting this association in the literature. $^{12}$ The issues raised about HCC following DAA therapy, which led to an avalanche of studies with contradictory findings, make these anecdotal observations more disquieting.

The suppression of HCV viral replication would allow, in most cases, a regression of HCV-related extrahepatic symptoms, and thus antiviral treatment should be considered without delay in patients with clinically significant extrahepatic manifrstations. However, as to the effect of antiviral therapy on lymphoproliferative disorders, data are still quite contradictory. Although treatment with interferon regimen was reported to reduce the risk of lymphoma, El-Serag et al have recently shown in a large cohort that successful DAA treatment resulting in SVR was not associated with significant risk reductions in nonHodgkin's lymphoma. ${ }^{3}$ Moreover, in terms of immune response, data showed that DAA treatment can be effective as to reducing the frequency of pathological $\mathrm{B}$ cells in the peripheral blood of HCV seropositive patients, but monoclonal populations can persist after viral eradication. ${ }^{4}$ Hence, this suggests that DAAs might have a lower antilymphoma activity than interferon therapy, which acts as an immunological modulator, explaining that the development of malignant lymphomas may occur after HCV clearance with the new drugs.

Besides, escaping from immune surveillance due to a decrease in virus-induced inflammation, as it has been hypothesised with HCC after DAA use, may lead to uncontrolled proliferation of malignant clone. Lymphomagenesis could be initiated by the relief of lymphoma cell inhibition from sustained HVC signal stimulation after viral clearance.

Moreover, DAA administration was associated with increased serum vascular endothelial growth factor (VEFG), and consequently, this may cause imbalance of the antitumor surveillance of the host. ${ }^{5}$ Interestingly, VEFG is also found to be overexpressed and to play a role in the progression of non-Hodgkin's lymphoma. ${ }^{6}$ This mechanism could be a rational of lymphomagenesis.

In our case, the diagnosis made immediately at the end of the treatment could be considered as a very short period for the development of hepatic lymphoma and may suggest unrecognised lymphoma. However, the fact that the patient was in a good condition and that there was no evidence of hepatic mass or malignancy on ultrasound just before the therapy suggests that hepatic lymphoma may be linked to the prescribed antiviral therapy. Currently, there is no clear evidence supporting the relationship between PHL and DAAs due to its rarity, and the possible mechanisms mentioned should be evoked and investigated in further studies.

Regarding the diagnosis of PHL, it may be challenging as it is not only an extremely rare tumour but also often misdiagnosed. Careful work-up and follow-up 
are mandatory to exclude primary disease elsewhere. Clinical manifestations, laboratory findings and imaging features are usually non-specific, making it difficult to distinguish from other more common hepatic tumours such as HCC, especially in a context of cirrhosis treated by DAAs, or metastatic disease when presenting with multiple nodules, as it is in our case. Thus, definitive diagnosis requires liver biopsy with immunohistochemical staining, which is essential for subtype determination, and this should be repeated if the first attempt is unremarkable.

In conclusion, we reported an extremely rare case of PHL occurring in patient with HCV-related cirrhosis shortly after viral clearance with sofosvubir and ribavirin. This report raises the issue about the possible development of hepatic lymphoid malignancy despite successful HCV treatment with DAAs. There is currently no clear evidence of the role of interferon-free antiviral treatment on hepatic lymphomagenesis; therefore, caution is needed before drawing final conclusions. Nevertheless, physicians should be aware of the risk during and after direct antiviral therapy for better monitoring, rapid diagnosis and early management.

Twitter Myriam Ayari @Myriam_Ayari

Acknowledgements We express our gratitude to the staff at Charles Nicolle Hospital who were involved in the patient care.

Contributors $\mathrm{YZ}$ and MA gathered the information, was involved in the definition of intellectual content and literature search, and wrote the manuscript. WC and MJ extracted and interpreted radiological data. FLK assisted in the preparation of the manuscript. SA and EBHM contributed to the critical review of the manuscript.

Funding The authors have not declared a specific grant for this research from any funding agency in the public, commercial or not-for-profit sectors.
Competing interests None declared.

Patient consent for publication Not required.

Ethics statements Written consent was obtained from the patient.

Provenance and peer review Not commissioned; externally peer reviewed.

Data availability statement All data relevant to the study are included in the article or uploaded as supplementary information. All data are available as part of the article and no additional source data are required.

Open access This is an open access article distributed in accordance with the Creative Commons Attribution Non Commercial (CC BY-NC 4.0) license, which permits others to distribute, remix, adapt, build upon this work non-commercially, and license their derivative works on different terms, provided the original work is properly cited, appropriate credit is given, any changes made indicated, and the use is non-commercial. See: http://creativecommons.org/licenses/by-nc/4.0/.

ORCID iD

Myriam Ayari http://orcid.org/0000-0001-7361-8248

\section{REFERENCES}

1 Andrade XA, Paz LH, Nassar Mo" ath, et al. Primary liver diffuse large B-cell lymphoma following complete response for hepatitis $\mathrm{C}$ infection after direct antiviral therapy. Acta Haematol 2018;139:77-80.

2 Shimagaki T, Maeda T, Kinjo N et al. Primary hepatic follicular lymphoma 5 years post sustained virological response from hepatitis C viral infection. J Clin Pathol 2021;74:e3.

3 El-Serag HB, Christie IC, Puenpatom A, et al. The effects of sustained virological response to direct-acting anti-viral therapy on the risk of extrahepatic manifestations of hepatitis $\mathrm{C}$ infection. Aliment Pharmacol Ther 2019;49:1442-7.

4 Schiavinato A, Zanetto A, Pantano G, et al. Polyclonal and monoclonal $\mathrm{B}$ lymphocytes response in $\mathrm{HCV}$-infected patients treated with direct-acting antiviral agents. J Viral Hepat 2017;24:1168-76.

5 Villani $R$, Facciorusso A, Bellanti F, et al. DAAs rapidly reduce inflammation but increase serum VEGF level: a rationale for tumor risk during anti-HCV treatment. PLoS One 2016;11:e0167934.

6 Yang J, Li W, He X, et al. Vegf overexpression is a valuable prognostic factor for non-Hodgkin's lymphoma evidence from a systemic metaanalysis. Dis Markers 2015;2015:1-9. 